\title{
A Complex Interplay: Cognitive Behavioural Therapy for Severe Health Anxiety in Addison's Disease to Reduce Emergency Department Admissions
}

\author{
Jo Daniels \\ Department of Psychology, University of Bath, Bath, UK \\ Elizabeth Sheils \\ Department of Psychology, University of Bath, Bath, UK
}

\begin{abstract}
Background: Addison's disease (AD) is a rare chronic illness caused by adrenocortical insufficiency. Due to the pivotal role of the regulating hormone cortisol in AD, there is a common symptom overlap between the presentation of anxiety and adrenal crisis. Previous literature has identified the prevalence of anxiety in endocrinological disorders, however there is a paucity of research examining the complex interplay between AD and anxiety. Aims: This paper describes a single case study of a patient with severe health anxiety and co-morbid AD. The aims of the study were to establish if standard cognitive behavioural therapy for health anxiety in AD can lead to a reduction in psychological distress, and whether this approach is an effective intervention for the reduction of Emergency Department admissions. Method: A single case design was used, with pre- and post-measures of health anxiety, general anxiety and depression. Data on Emergency Department admissions prior to and following treatment were used to assess change in this domain. Results: Reliable and clinically significant reductions were seen across all measures, from severe to sub-clinical levels. There was a complete amelioration of Emergency Department admissions in the 12 months following completion of treatment. Conclusions: This preliminary study provides a sound rationale for further research into AD complicated by anxiety. Findings support the clinical utility of the cognitive behavioural therapy model for complex presentations of $\mathrm{AD}$, offering a potential treatment option where anxiety is elevated and interfering with self-management and leading to high levels of health service use.
\end{abstract}

Key words: Addison's disease, cognitive behaviour therapy, Emergency Department

\section{Introduction}

Addison's disease (AD) is a rare autoimmune disease occurring as a result of adrenal insufficiency. The condition causes symptoms of fatigue, dizziness, weakness, vertigo, changes in weight and pigmentation of the skin (Gance-Cleveland, 2003). The condition is potentially life threatening due to the possibility of adrenal crisis, caused by significantly low levels

Correspondence to Dr Jo Daniels, University of Bath, Department of Psychology, Claverton Down, Bath BA2 7AY, UK. E-mail: j.daniels@bath.ac.uk 
of cortisol. Treatment takes the form of synthetic corticosteroids or adrenal replacement (Gance-Cleveland, 2003), which involves constant monitoring and titration of medication. The treatment regime for AD is often tightly controlled and due to individual differences and the variable influence of stress and illness on $\mathrm{AD}$, management of the disease is complex.

In addition to the biological factors seen in $\mathrm{AD}$, psychological factors such as cognitive processing and interpretation of stimuli (e.g. misperception of threatening information) can also activate the hypothalamic-pituitary-adrenal (HPA) axis and precipitate activation of the peripheral nervous systems (Kim and Gorman, 2005), also known as 'fight or flight'. Chronic misinterpretation of stimuli presents a complex clinical and personal challenge in AD, as HPA activity and sustained anxiety can mimic AD symptoms such as palpitations, dizziness and changes in bodily sensations. The presence of changes in the HPA may have implications for the development of affective disorders; however, there is little research into the psychological aspects of autoimmune conditions, or the interplay between anxiety/stress and AD. This is surprising given the pivotal role of cortisol and the HPA axis in both of these conditions.

The cognitive model of health anxiety (Salkovskis, 1997) suggests that prior beliefs about illness influence the interpretation of symptoms and can lead to misinterpretations about 'normal' bodily variations. For AD and co-morbid anxiety, the picture is complicated by the necessary early detection of infection and illness in managing $\mathrm{AD}$; however, strategies such as symptom hypervigilance (commonly seen in health anxiety) are counterproductive and can elevate and intensify symptomatic experience of potentially innocuous bodily variations. Due to the implications of positively detecting illness/infection, an anxiety/stress response is likely to be triggered, which then activates the HPA, leading to further symptoms that can be misinterpreted as confirmation of the presence of illness or AD crises - and the anxiety cycle continues.

Due to the life-threatening nature of AD, the Emergency Department (ED) may be considered an appropriate place to present during an adrenal crisis; however, where severe health anxiety and $\mathrm{AD}$ present in combination, attendances may be precipitated by peaks in anxiety, rather than an adrenal crisis. The dilemma for the patient is that the two may feel indistinguishable, and due to the prevailing levels of anxiety the ED may feel like a 'safe' place to be. This further reinforces over-reliance on the ED as a coping strategy, rather than more effective self-management strategies that are not fuelled by anxiety.

\begin{abstract}
Aim
The aim of this study was to establish whether a standard treatment for health anxiety can lead to a reduction in psychological distress and ED admissions for a patient with AD. The secondary aim was to facilitate the development of an individualized AD self-management plan for the patient, who we will refer to as K.L.
\end{abstract}

\title{
Method
}

To achieve the primary aim of the study, an $A B$ case study design was utilized to explore the effectiveness of cognitive behavioural therapy (CBT) for health anxiety in a case of AD. Treatment consisted of twelve 60-minute sessions of CBT for health anxiety (as described by Salkovskis et al., 2003) and was delivered by a chartered clinical psychologist accredited 
in cognitive behavioural psychotherapy (British Association for Behavioural and Cognitive Psychotherapies, BABCP) with specialist expertise in treating health anxiety in health settings.

To achieve the secondary aim of developing an individualized self-management plan, K.L.'s endocrinologist and general practitioner were contacted via telephone for a brief consultation. This measure was taken to ensure that the self-management plan was both appropriate to medical need and also represented a shared understanding of the problem and management plan.

K.L. was treated in a clinic attached to an acute hospital ED, specifically set up to work with patients who frequently attended the ED. K.L. was aware of the referral from the ED to the clinic and consented to participate in an experimental study specifically focused on both reducing psychological distress and ED attendance.

\section{Measures}

The Health Anxiety Inventory (HAI), Patient Health Questionnaire (PHQ-9) and Generalized Anxiety Disorder (GAD-7) measures were used pre- and post-treatment. These standardized measures were deemed valid and reliable. Data on ED admissions were taken for the 12 months preceding and following the course of therapy and were acquired through hospital business intelligence.

\section{Case}

K.L. was a single female in her 40s who had been diagnosed with AD twenty years earlier. She presented as very warm and engaging, offering a high degree of insight and psychological awareness in early sessions. She reported significant levels of anxiety related to her condition, regularly attending the ED when she suspected an AD crisis. She attended the ED as a measure to prevent worsening of her condition, and considered the ED to be a 'safe' place. 'Signs' of a suspected AD crisis included changes in bodily variations accompanied by feeling generally unwell. K.L. reported that around 50\% of ED attendances resulted in a positive diagnosis of an underlying infection.

K.L. presented as very emotionally expressive, however she perceived this to be a 'weakness' particularly in comparison with her family whom she felt 'controlled' their emotions.

An episode of health anxiety would usually be triggered by a change in bodily sensations or exposure to an illness, e.g. a virus and would lead to negative thoughts relating to her feared worst case scenario: her family abandoning her while critically ill (to die alone) in the ED. According to K.L. an adrenal crisis or other significant illness would inevitably reveal her 'unloveable weakness' and reflect her personal failure in managing her physical and emotional health. It was hypothesized that this was the result of a combination of early life experiences, e.g. not feeling accepted by her family, emotion being perceived as weakness which was then compounded by a difficult to manage condition that elevated health related anxiety. K.L. reported struggling on a daily basis to manage her complex health condition and the significant worry about the potential implications of her condition.

K.L. employed a number of behavioural strategies that were consciously directed at preventing or halting the progression of illness, ultimately to keep her 'safe' from abandonment. These were habitual body scanning, vigilance to bodily sensations, checking behaviours, thought control, delaying medical assistance, controlled breathing, reassurance seeking and 


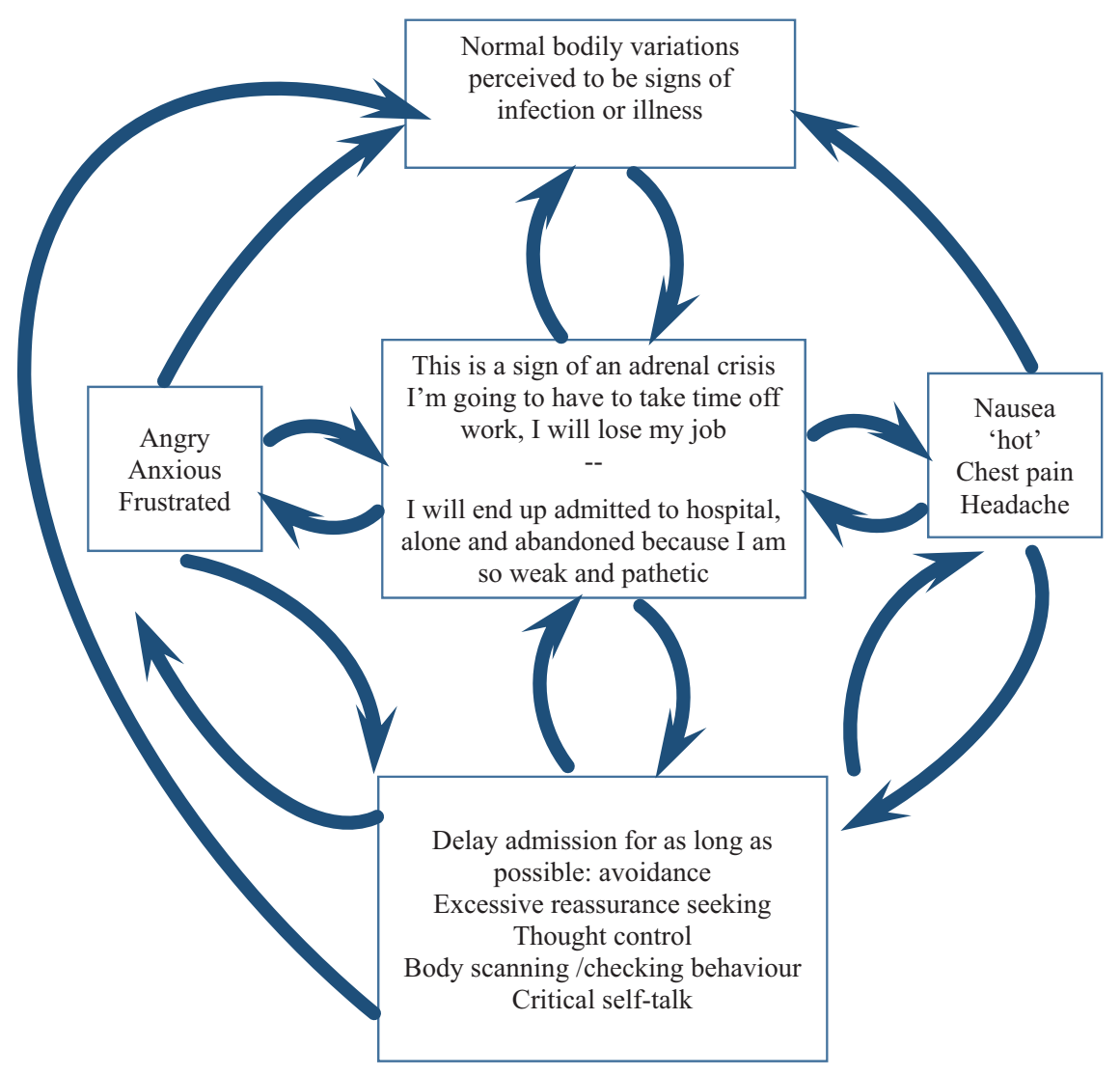

Figure 1. (Colour online) Cognitive behavioural formulation of presenting difficulties

critical self-talk. Vicious cycles formed as these behaviours served to heighten intensity of physiological sensations, increase anxiety, and generate 'evidence' for the underlying threat beliefs that she was both unwell and 'weak' while allowing no opportunity to disconfirm her beliefs (see Fig. 1), which resulted in significant psychological distress and heightened anxiety.

K.L. indicated that the ED represented a place of safety for her, but also a source of fear. K.L. believed that as long as she was in the ED or indeed admitted, that 'threat to her life' diminished; however, she found the fear of abandonment triggered by attending the ED (and the perceived implication of 'weakness/failure') emotionally intolerable. This represented a complex dilemma for K.L. who felt compelled to choose between her physical and emotional wellbeing.

\section{Treatment}

K.L. identified her primary difficulty as 'anxiety' and the impact and interaction of this on her ability to confidently and effectively manage her condition. Furthermore, K.L. expressed concern about her worry and preoccupation with physical symptoms, reporting that she often feared catastrophic outcomes, e.g. death, which caused her great distress. 
K.L.'s clinical presentation and scores on the HAI ( $>18)$ were consistent with a diagnosis of health anxiety. CBT for health anxiety was therefore considered to be the most appropriate intervention to address K.L.'s presenting difficulties. Health anxiety and the associated CBT were explained to the patient who consented to treatment.

The initial stages of therapy were driven by developing a shared collaborative understanding of K.L.'s presenting difficulties and socializing her to the model (Roos and Wearden, 2009) of CBT for health anxiety. Similarities between anxiety and AD symptoms were explored, revealing that physical sensations could be misinterpreted as signs of infection or AD rather than normal bodily variations or anxiety. Further discussion and body focus tasks elicited the possibility that these misinterpretations may be triggering counter-productive behaviours that served to increase her anxiety and physical symptoms.

The 'Theory A vs Theory B' approach facilitated discussions regarding the possibility of an alternative explanation for changes in bodily variations. K.L. engaged well with the idea of taking a scientific approach to generate evidence to support either theory. The theories were:

Theory A: I need to take measures to prevent an AD crisis, and if I do not prevent an adrenal crisis then I will become critically ill and die alone.

Theory B: I am taking measures to prevent an $\mathrm{AD}$ crisis; however, these measures may be contributing to or maintaining the problem, rather than helping.

Treatment proceeded based on the agreement that sessions and inter-session work would be used to generate evidence to test theory A or B. Cognitive strategies were used to operationalize and challenge underlying beliefs such as 'If I am ill then I am weak, pathetic, a failure'. K.L. was able to identify that her reassurance seeking, expectation of others' and 'mind reading' may have maintained fears of abandonment. Verbal reattribution techniques such as pie charts were used to illustrate alternative explanations for physical sensations, particularly 'palpitations' and feeling 'hot'.

A Socratic approach was used to examine the behaviours employed by K.L. to manage her symptoms with a view to establishing whether they were helpful or unhelpful, particularly given the reciprocal relationships between her strategies and the impact on affect and physiology.

Behavioural experiments were used to test the usefulness of these strategies, centred on manipulating safety behaviours and observing outcomes. K.L. generated evidence that highlighted that not only were her strategies unhelpful, but also that the strategy of delaying admission could potentially increase the risk of adverse consequences in the event of a crisis. K.L. was able to identify that due to the self reinforcing nature of the strategies and subsequent elevation of symptoms, she was unable to reliably distinguish between normal bodily variations, anxiety, and $\mathrm{AD}$ associated symptoms, therefore focusing on bodily sensations did not generate helpful evidence.

The use of ED as a 'place of safety' was explored using verbal reattribution and was then re-conceptualized. Through discussion it was evident that the ED represented the endpoint in a vicious cycle of heightened health anxiety, rather than a place of physical or emotional safety. K.L. shifted to a position of viewing ED admissions as the result of sub-optimal self-management and that through development of an improved and more effective selfmanagement plan, ED admissions could be avoided on most, if not all, occasions. Final sessions focused on reviewing evidence in favour of theory A or B, and developing coping strategies that were more likely to be helpful and promote self-management, prevent hospital admission, and 
enable her to have a clearer insight into her difficulties. These strategies informed the therapy blueprint and were developed with K.L.'s endocrinologist and general practitioner, resulting in a management plan that could be supported and consistently implemented.

The strategies were spontaneously tested towards the end of therapy, when a suspected adrenal crisis was successfully self-managed at home in a timely way that was less distressing. Changes in self-management behaviours included shifting focus away from symptoms, adopting more self-reliance rather than reassurance seeking, and requesting advice from a general practitioner rather than actively avoiding medical assistance.

\section{Results}

Pre- and post-measures indicated a reduction from severe to sub-clinical range across all measures (HAI: 31 to 12; GAD-7: 17 to 2; PHQ-9: 19 to 3). Reliable Change Index analysis reflected that reductions in scores were reliable and clinically significant. Data from the weekly administration of the HAI reflected a clear downward trajectory week by week, with the exception of an unexpected 'spike' into the clinical range at session 8 before returning to sub-clinical levels at session 9.

At end of therapy, K.L. reported increased confidence in successfully managing health concerns using the new management plan and reported a complete abandonment of her reliance on the ED as a 'safe' place. Further follow-up data were not available.

In the 12 months prior to therapy, K.L. was admitted on six occasions. For the 12 months following therapy, K.L. was not admitted to the ED.

\section{Discussion}

This study reports preliminary evidence for the clinical utility of a standard CBT treatment for health anxiety to reduce ED admissions and psychological distress in AD. Results reflect reliable and clinically significant changes from case to non-case status across three standardized measures and a complete extinction of ED admission during the 12 months following completion of therapy. Based on K.L.'s anecdotal evidence that $50 \%$ of her presentations at ED indicated the presence of an infection, we tentatively suggest that admission data may reflect a successful implementation of her self-management plan, whereby she no longer delayed accessing general practitioner input which resulted in ED admission, but alternatively sought advice earlier and self-managed in the community.

Due to the critical nature of the threat of adrenal crisis, it is understandable that AD patients may be cautious and vigilant around bodily changes, leading to a susceptibility to healthfocused anxiety cycles reported to be common in other medical conditions (Tyrer et al., 2014). The AD patient is placed in a continuously precarious position when differentiating normal bodily variations, signs of illness and psychophysiological symptoms of anxiety, which forms both a personal challenge to the patient and a unique clinical challenge to the treating clinician. It may be useful for clinicians working with $\mathrm{AD}$ and related conditions to consider brief screening measures such as those used in this study, to ensure appropriate management strategies encompass the potential confounding effects of anxiety occurring with $\mathrm{AD}$. In the case where anxiety occurs in a way that complicates or obstructs treatment, or causes high levels of distress, CBT for health anxiety should be considered as a treatment option. 
This treatment intervention offers an example of successfully integrating both physical and mental health care, which is in line with recommendations made by the King's Fund (Naylor et al., 2016) and NHS England (2016). Since completion of this case, Improving Access to Psychological Therapies (IAPT) have evolved to include long-term conditions, with the aim of making evidence-based interventions such as the one described in this paper accessible to patients with both physical and mental health needs. This paper offers preliminary evidence of the applicability of integrated physical and mental health care for AD and offers an example of multi-disciplinary collaboration to enhance and promote more effective long-term selfmanagement. Consultation of trusted practitioners involved in K.L.'s long-term and routine care appeared to increase confidence in the self-management plan both on the part of the patient and therapist, perhaps offering a sense of medical 'safety' that replaced the ED. This should be considered in complex case treatment with co-morbidities, particularly due to the low resource demands and potentially high impact.

The present study is preliminary in nature and has noted limitations: the case was not a planned single case experimental design and therefore multiple baseline measures were not available to confirm a stable presentation of health anxiety. Frequent admission to the ED in the preceding months is suggestive of a history of health anxiety; however, this is speculative.

This study provides a sound rationale for further research and clinical focus on health anxiety in $\mathrm{AD}$, a complex clinical presentation with significant personal and healthcare economic implications.

\section{Acknowledgements}

The authors would like to thank Dr Mike Osborn and Professor Paul Salkovskis for their respective advice on this case, and of course patient K.L.

Financial support: None.

Conflicts of interest: Jo Daniels and Elizabeth Sheils have no conflicts of interest with respect to this publication.

Ethics statement: The authors have abided by the Ethical Principles of Psychologists and Code of Conduct as set out by the APA. Ethical approval was not necessary as the case study was a standard application of routine clinical practice.

\section{References}

Gance-Cleveland, B. (2003). Adaptation to Addison's disease in a child: a case study. Journal of Pediatric Health Care, 17, 301-310.

Kim, J. and Gorman, J. (2005). The psychobiology of anxiety. Clinical Neuroscience Research, 4, 335-347.

Naylor, C., Das, P., Ross, S., Honeyman, M., Thompson, J. and Gilburt, H. (2016). Bringing together physical and mental health: a new frontier for integrated care. Retrieved from: https://www.kingsfund. org.uk/sites/files/kf/field/field_publication_file/Bringing-together-Kings-Fund-March-2016_1.pdf (accessed 16 March 2017). 
NHS England Mental Health Task Force (2016). Implementing the five year forward view for mental health. NHS England. Retrieved from: https://www.england.nhs.uk/wp-content/uploads/2016/ 07/fyfv-mh.pdf (accessed 16 March 2017).

Roos, J. and Wearden, A. (2009). What do we mean by 'socialization to the model'? A Delphi Study. Behavioural and Cognitive Psychotherapy, 37, 341-345.

Salkovskis, P. M. (ed). (1997). Frontiers of Cognitive Therapy. Guilford Press.

Salkovskis, P. M., Warwick, H. and Deale, A. C. (2003). Cognitive-behavioral treatment for severe and persistent health anxiety (hypochondriasis). Brief Treat Crisis Intervention, 3, 353.

Tyrer, P., Cooper, S., Salkovskis, P., Tyrer, H., Crawford, M., Byford, S. et al. (2014). Clinical and cost-effectiveness of cognitive behaviour therapy for health anxiety in medical patients: a multicentre randomised controlled trial. Lancet, 383, 219-225. 